\title{
PARITY AND GRAVIDITY AS CORRELATES OF MATERNAL MORTALITY IN EKITI STATE, NIGERIA.
}

\author{
Fabamise Olufemi Moses ${ }^{1 *}$, O. Iluku-Ayoola ${ }^{2}$, Olowoyeye Ebenezer Adeola ${ }^{3}$ \\ *1,2,3 Department of Basic Medical Sciences, College of Health Sciences \& Technology, Ijero - Ekiti. Ekiti State, \\ Nigeria.
}

corresponding author:

Fabamise Olufemi Moses ${ }^{1}$

${ }^{1,2,3}$ Department of Basic Medical Sciences, College of Health Sciences \& Technology, Ijero - Ekiti. Ekiti State, Nigeria. femibamise@gmail.com.

\begin{abstract}
: -
This study investigated parity and gravidity as correlates of maternal mortality in Ekiti State. It critically and explicitly examined the extent to which parity and gravidity stands as correlates of maternal mortality in Ekiti. In view of these, the study adopted descriptive research design of the survey type which comprises of all the Doctors and Nurses who attend to pregnant women during pregnancy, delivery and after delivery. Simple random sampling technique was used to select 550 doctors and nurses from the twenty (20) general and specialist hospitals in Ekiti State. A well-structured questionnaire was used as instrument for data collection. The data collected was analyzed using statistical Analysis System (SAS) mode. Pie chart was used to provide answers to the research questions and inferential statistics of Pearson Product Moment Correlation was used to test the hypotheses at 0.05 level of significance. The results showed that parity and gravidity have high effect on maternal mortality and significantly correlate to maternal death in Ekiti state. Hence, more awareness should be created on the frequency of pregnancy and birth by the government, NGOs and other health regulated agencies, hence reducing this menace of maternal death. It is also recommended that women should have an interval of at least 24 months from a live birth to the next pregnancy in order to reduce maternal mortality.
\end{abstract}

Keywords: Parity, Gravidity, maternal mortality

\section{(a) $(\$)$}




\section{INTRODUCTION}

Maternal mortality has been observed to be one of the major causes of death among women of reproductive age in many countries and remains a serious public health issue especially in developing countries. Maternal mortality refers to deaths due to complications from pregnancy or childbirth. (Shah \& Say 2007). The global maternal mortality ratio as reported by the United Nation inter-agency (2013) declined by 45 percent from 380 deaths to 210 deaths per 100,000 live births. This translates into an average annual rate of reduction of $2.6 \%$. While this may look impressive, it is less than half the $5.5 \%$ rate needed to achieve the three-quarters reduction in maternal mortality targeted for 2015 of the fifth Millennium Development Goal (MDG) which could not be achieved before the expiration of the MDG.

The issue of maternal deaths emerged as a world health concern through the launching of the Safe Motherhood Initiative (SMI) by the United Nations in Kenya in 1987 and in Nigeria in 1990. The target of the Safe Motherhood Initiative programme was to reduce maternal mortality figure of 500,000 by 50 percent by the year 2000 this also was not also achieved. Generally, the realization of low maternal death may remain a mirage in the world and specifically in Nigeria if concrete measures are not taken. Maternal mortality is observed to be unacceptably high. About 830 women die from pregnancy- or childbirthrelated complications around the world every day. The World Health Organisation, WHO (2015) projected that roughly 303,000 women may die during and following pregnancy and childbirth and that almost this death which can be prevented would occur in low resource settings.

It appears that some of the contributory factors to maternal mortality in Nigeria include malnutrition, poor environmental hygiene, low access and utilization of quality health care services by women and literacy level. Some women seem to be more at risk than others in Nigeria. For instance, the rural dwellers due to their less privilege and access to medical services may be more prone to complication and situation that leads to maternal mortality. Inability to have prompt treatment for some medical cases such as ante partum or post-partum hemorrhage, post-delivery infection, hypertensive disorders in pregnancy and obstructed labour may predispose women to death.

\section{Parity and Maternal Mortality}

Emilly,Willyane \& John, (2013) affirmed the fifty year " maternal depletion syndrome" which is as a result of the physical and caloric demands of repeated pregnancy, in combination with the physical and caloric stresses of life at a subsistence level. In the fifty years since the origin of this theory, debate continues as to whether parity is positively associated with outcomes such as anemia, low maternal weight gain, and low-birth weight, given confounding factors such as maternal age, the health status of mothers that are able to achieve high parity, breastfeeding duration, the spacing between pregnancies, changes in body fat distributions through successive pregnancies, and rapidly shifting diets across the globe( Adebowale, Adepoju \& Fagbamigbe, 2011).

A theory also focuses principally on poorer nutritional status of high parity children as a result of lesser parental investment and/or competition between siblings for finite resources (Leight,2010). However, proving these correlations is complicated by the need to account for household composition such as the child-dependency ratio within the household, the age and gender of the person heading the household, and the presence of twins or triplets. It could be assumed that high parity women are overall less likely to access health services for themselves or their children, due to a strain on resources, the inability to find time given the need to care for so many children, or a reduced sense of urgency as pregnancies and childhood illnesses become repetitive. It could also be that lack of physical access to health facilities might be a barrier to accessing family planning services which could lead to more higher parity births.

\section{Gravidity and Maternal Mortality}

In poorer and richer countries, adverse birth outcomes are associated with short birth intervals (Cleland, CondeAgudelo \& Peterson, 2012). The WHO (2007) recommends an interval of at least 24 months from a live birth to the next pregnancy. Inter-pregnancy or inter-birth intervals have additionally been associated with short-term maternal outcomes. Lack of sufficient time to return to the normal pre-pregnancy metabolic state before the next pregnancy may also initiate processes with longer term implications; for example, a prospective US study found that risks of maternal obesity increased with each inter-pregnancy interval of less than 12 months (Davis, Babineau \& Wang, 2014).

The physical, emotional, and economic strains involved in meeting the needs of two or more children close in age may also lead to stresses relevant to health. Mothers of twins, for example, suffer from higher rates of postnatal depression than mothers of singletons (Jena, Goldman \& Joyce, 2011). Given the importance of accumulated life course effects on health in later life, stresses associated with short inter-birth intervals may have longer term health implications for mothers.

The World Bank (2008) reported that $51.6 \%$ of Nigerians live in rural areas, most of whom are cut off from modern medical facilities, making pregnant women vulnerable to preventable adverse outcomes. Most of these adverse outcomes result from delay in seeking care, getting to health centers when care is sought, receiving care on getting to the health centre, and referring patients to more advanced centers when necessary. It seems the problem of poor organization and access to maternal health services is a major challenge in Nigeria.

The issue of maternal mortality in Nigeria has been adduced to both medical and social factors and it is believed that the way to take maternal mortality is to simultaneously deal with these factors. However, while numerous studies have focused on the medical factors that cause maternal mortality, few had worked on parity and gravidity as it relates to maternal mortality especially in Ekiti State. This study therefore examined parity and gravidity as correlates of maternal mortality in Ekiti state. 


\section{Statement of the Problem}

It has been observed by the researcher that, in spite of all the policies, declarations, conferences and other efforts aimed at reducing the scourge of maternal deaths across the globe, only modest gains in maternal mortality reduction appear to have been achieved in many countries including Nigeria. Although maternal deaths in Nigeria may be observed to be mainly due to complications of pregnancy and delivery, the socio-cultural factors such as parity and gravidity, under which these pregnancies and deliveries occur may pave way for complications and deaths. Incidentally very few studies have focused exclusively on this aspect. This study therefore examined parity and gravidity as correlates of maternal mortality in Ekiti State.

\section{Purpose of the Study}

The purpose of this study was to examine parity and gravidity as correlates of maternal mortality in Ekiti. It considered the extent to which parity and gravidity relate to maternal mortality in Ekiti State

\section{Research Questions}

The following research questions were raised for the study:

1. To what extent does parity relate to maternal mortality in Ekiti State?

2. To what extent does gravidity relate to maternal mortality in Ekiti State?

\section{Research Hypotheses}

The following hypotheses were tested.

1. Parity will not be a correlate of maternal mortality in Ekiti state.

2. Gravidity will not be a correlate of maternal mortality in Ekiti state.

\section{Significance of the Study}

The study would be of great benefit to mothers, health workers, hospital administrators, Government and NonGovernmental Organisations (NGO). Findings from this study could provide information on parity and gravidity as factors that could lead to maternal mortality. Understanding and identification of parity and gravidity as factors that affect maternal mortality will be of importance to health educators because it will help in designing health education and health promotion programmes to provide right information on maternal mortality during antenatal and postnatal care. It is believed that the outcome of this study will benefit primary health workers to disseminate the right information on maternal health, hence reducing mortality. Finally, this study may also be useful to all stake holders in health sectors, especially non-governmental organizations (NGOs) and policy makers when planning and monitoring health programmes related to maternal health.

\section{Methodology}

The descriptive research design of the survey type was adopted for the study. It comprises of all 654 health personnel (Doctors and Nurses) in the seventeen (17) general and three (3) specialist hospitals in Ekiti State. The doctors (60 of them) were purposively and proportionately selected from the population while simple random sampling techniques was adopted in selecting 490 Nurses, making a total of 550 samples. A self-structured questionnaire titled: Causes of Maternal Mortality (CMM) was used for data collection which were analysed using charts (Pie chart) and inferential statistics of Pearson Product Moment Correlation (PPMC) was also used to test the hypotheses at 0.05 level of significance.

\section{Results and Discussion \\ Descriptive Analyses \\ Question 1: To what extent does parity relates to maternal mortality in Ekiti State?}

\section{Gravidity as correlate of Maternal Mortality}

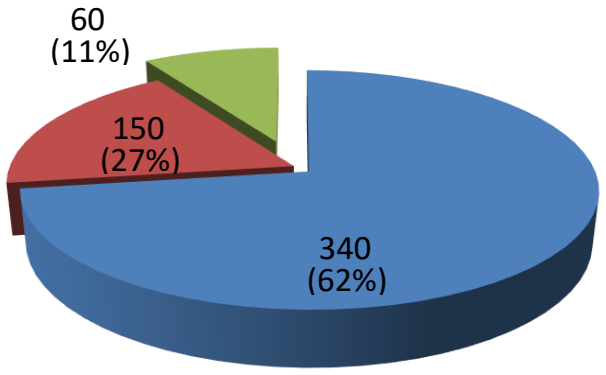


Figure 1 revealed the extent to which parity contributes to maternal mortality in Ekiti State. It was revealed that out of 550 respondents sampled for the study, 340 representing $62 \%$ indicated that parity contributed to high extent on maternal mortality in Ekiti State. Question 2: To what extent does gravidity relate to maternal mortality in Ekiti State?

\section{Gravidity as correlate of Maternal Mortality}

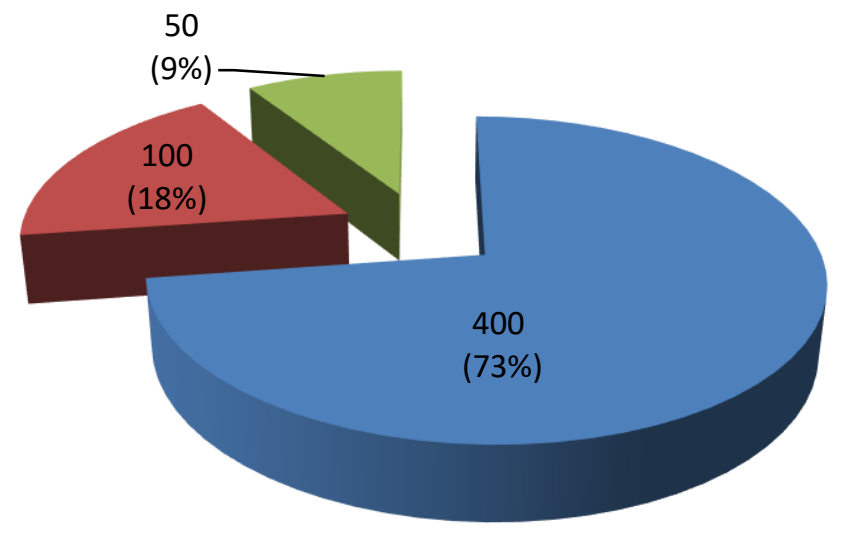

Figure 2: Pie Chart showing the extent to which gravidity contributes to Maternal Mortality

The pie chart in figure 2 revealed the extent to which gravidity contributes to Maternal Mortality. It was revealed that out of 550 respondents, $400(73 \%)$ indicated that gravidity contributed to a high extent on maternal mortality in the Ekiti state.

Hypothesis 1: Parity will not be a significant correlate of maternal mortality in Ekiti state.

Table 1: Relationship between parity and maternal mortality in Ekiti State

\section{$\mathbf{P}<0.05 *$ Significant}

\begin{tabular}{|l|l|l|l|l|l|l|}
\hline Variable & N & Mean & SD & r-cal & r-tab & Sig (P) \\
\hline Parity & 550 & 2.89 & 0.63 & & & \\
\cline { 1 - 1 } $\begin{array}{l}\text { Maternal } \\
\text { Mortality }\end{array}$ & 550 & 2.76 & 0.74 & $0.50^{*}$ & 0.23 & 0.01 \\
\hline
\end{tabular}

The result of analysis presented in table 1 revealed that there was high relationship between parity and maternal mortality in Ekiti state as $r_{\text {cal }}(0.50)$ was greater than $r_{\text {tab }}(0.23)$ and $P$-value (0.01) less than 0.05 level of significance. This led to the rejection of hypothesis one. Hence, parity is a significant correlate of maternal mortality in Ekiti state.

Hypothesis 2: Gravidity will not be a significant correlate of maternal mortality in Ekiti state.

Table 2: Relationship between gravidity and maternal mortality in Ekiti State

\begin{tabular}{|l|l|l|l|l|l|l|}
\hline Variable & N & Mean & SD & r-cal & r-tab & Sig (P) \\
\hline Gravidity & 550 & 2.61 & 0.72 & & & \\
\cline { 1 - 4 } Maternal Mortality & 550 & 2.76 & 0.74 & $0.55^{*}$ & 0.23 & 0.02 \\
\hline
\end{tabular}

$\mathbf{P}<0.05 *$ Significant

The result of analysis presented in table 2 revealed that there was high relationship between gravidity and maternal mortality in Ekiti state as $r_{\text {cal }}(0.55)$ was greater than $r_{\text {tab }}(0.23)$ and P-value (0.02) less than 0.05 level of significance. This led to the rejection of hypothesis two. Hence, gravidity is a significant correlate of maternal mortality in Ekiti state.

\section{Discussion}

Parity was revealed as a correlate of maternal mortality in Ekiti state. This finding corroborated with the finding of Leight (2010) that high parity women are overall less likely to access health services for themselves or their children. Low parity woman has the ability to find time given the need to care for so many children, or a reduced sense of urgency for pregnancies and childhood illnesses become avoidable. 
Gravidity was also revealed in the study as a correlate of maternal mortality in Ekiti state. Lack of sufficient time to return to the normal pre-pregnancy metabolic state before the next pregnancy may also initiate processes with longer term implications. Davis, Babineau, Wang (2014) found that risks of maternal obesity increased with each inter-pregnancy interval of less than 12 months. The WHO (2007) recommends an interval of at least 24 months from a live birth to the next pregnancy in order to reduce maternal mortality.

\section{Conclusions and Recommendations}

Based on the findings of the study, it was concluded that most maternal mortality cases in Ekiti state are as a result of number of time women give birth (parity) and number of time women get pregnant (gravidity). The study therefore recommends that more awareness should be created on the frequency of pregnancy and birth by the government, NGOs and other health regulated agencies, hence reducing this menace of maternal death. It is also recommends that women should have an interval of at least 24 months from a live birth to the next pregnancy in order to reduce maternal mortality.

\section{References}

[1].Adebowale, S.A , Adepoju O.T.\& Fagbamigbe, F.A. (2011). Child spacing and parity progression: Implication for maternal nutritional status among women in Ekiti communities, southwestern Nigeria. Pakistan Journal of Nutrition, $10(5), 485-491$.

[2].Cleland, J.,Conde-Agudelo, A.\& Peterson H. (2012). Contraception and health. Lancet, 380,149-156.

[3].Davis, E.M., Babineau D.C. \& Wang, X. (2014). Short inter-pregnancy intervals, parity, excessive pregnancy weight gain and risk of maternal obesity. Maternal Child Health Journal,18,554-562.

[4].Emily, S., Willyanne, D. P. \& John S. ( 2013). Linking high parity and maternal and child mortality: what is the impact of lower health services coverage among higher order births? BMC Public Health.13( 3) S7

[5].Jena, A.B., Goldman, D.P. \& Joyce G. (2011).Association between the birth of twins and parental divorce. Journal of Obstetrics Gynecology, 117,892-897.

[6].Leight J. (2010). Sibling rivalry: Ability and intra-household allocation in Gansu Pronvince China. University of Pennsylvania

[7].National Population Commission (NPC) [Nigeria] and ICF Macro (2009).

[8].Nigeria demographic and health Survey 2008. Abuja, Nigeria: National Population Commission and ICF Macro Ogujuyigbe, P. O. \& Liasu, A. (2007) Perception and Health-seeking behaviour of

[9].Nigerian women about pregnancy-related risks: strategies for improvement. Journal of Chinese Clinical Medicine, 2 (11).643-654.

[10]. Oladapo, O.T., Sule-Odu, A.O., Olatunji, A.O. \& Daniel OJ (2005). "Near-miss" obstetric events and maternal deaths in Sagamu, Nigeria: a retrospective study. Reproductive Health,2,(9) 2-9

[11]. Shah, I.H. \& Say L,. (2007) Maternal mortality and maternity care from 1990- 2005: Uneven but Important Gains Reproductive Health Matters, Maternal Mortality and Morbidity: Is Pregnancy Getting Safer for Women? Reproductive Health Matters, 15,(30)17-27

[12]. United Nation Inter-Agency (2013). Trends in maternal mortality: 1990 to 2013. Estimates by WHO, UNICEF, UNFPA, The World Bank and the United Nations Population Division. Geneva:WHO

[13]. WHO (2007). Maternal mortality in 2005; Estimates developed by WHO, UNICEF, UNFPA and The World Bank.Geneva.

[14]. World Health Organization, (2008). Making pregnancy safer: Maternal mortality and morbidity case review. Retrieved from; http//www.euro.who.int/infopages. August, 2008. 